\title{
A Smart Robot Training Data Acquisition and Learning Process Recording System Based on Blockchain
}

\author{
Kun Wang, Chen Yang, Tao Wang \\ The College of Nuclear Technology and Automation Engineering, Chengdu University of Technology, Chengdu, China \\ Email: 1213737146@qq.com,604493732@qq.com,wangt@cdut.edu.cn
}

How to cite this paper: Wang, K., Yang, C. and Wang, T. (2020) A Smart Robot Training Data Acquisition and Learning Process Recording System Based on Blockchain. Open Access Library Journal, 7: e6711. https://doi.org/10.4236/oalib.1106711

Received: August 12, 2020

Accepted: August 28, 2020

Published: September 1, 2020

Copyright $\odot 2020$ by author(s) and Open Access Library Inc.

This work is licensed under the Creative Commons Attribution International License (CC BY 4.0).

http://creativecommons.org/licenses/by/4.0/

\section{(c) (i) Open Access}

\begin{abstract}
The Internet of Things and artificial intelligence have developed rapidly in recent decades, and intelligent robots have been used in various fields of production and life. There are many requirements for the functions, performance and intelligence of smart robots. Many researchers are committed to how intelligent robots can learn more efficiently and develop new functions. How to obtain the learning data while protecting the privacy of users and how to record the learning process are two major problems faced by researchers. This article proposes a smart robot training data acquisition and learning process recording system based on blockchain to ensure that the training information of robot is kept credible and to provide users with privacy protection.
\end{abstract}

\section{Subject Areas}

Artificial Intelligence, Information and Communication: Security, Privacy, and Trust

\section{Keywords}

Smart Robot, Learning Data, Blockchain, Privacy

\section{Introduction}

With the rapid development of information technology, smart robots have become a reality [1]. AI-enabled robots can manipulate data received from their environment and can deal with situations such as humans [2]. As companies continue to invest in smart cities and contribute to the development of the Internet of Things, the Internet of Things provides huge opportunities for the 
growth of the smart robot market. The learning process of the robot is inseparable from the acquisition of learning materials and the recording of learning records. The design of advanced smart robots is becoming more and more complex, which poses a challenge to the safety performance of the robot. How to obtain the learning data while protecting the privacy of users and how to record the learning process are two major problems faced by researchers [3].

Blockchain originated from Satoshi Nakamoto's Bitcoin [4]. Blockchain is the core technology of cryptocurrency. More and more records, called blocks, are linked using encryption technology. Each block contains a cryptographic hash of the previous block, timestamp and transaction data which is usually expressed as the root hash of the Merkle tree [5]. The basic structure of blockchain is described in Figure 1. Blockchain has become a research hotspot because of its advantages such as anonymous, verifiable and tamper-proof trading history, and these characteristics can protect against any party's mistakes [6]. Many researchers [7] [8] [9] [10] explore the potential of distributed ledger technology, even though this is a young technology. Blockchain has been used in many fields such as traceability privacy protection [11]. In this paper, we proposed a blockchain-based smart robot training framework, which can protect the privacy of data producers and ensure that the robot learning information is truthfully preserved.

\section{The Proposed Blockchain-Based System}

The proposed system entities based on blockchain includes robot manufacturers, training platforms, blockchain platforms, and users. Figure 2 shows the system model.

Manufacturers produce robots and sell them to users. The attributes of robots include robot type, robot ID. Manufacturers can send tasks and rewards to the training platform to obtain data, including specified scene data and performance indicators of the user's robot.

The training platform can be a manufacturer or a third-party trusted platform. The training platform obtains tasks from manufacturers, assigns tasks to users, and regularly obtains data from the blockchain and sends them to manufacturers. The training platform get handling fee from it.

The blockchain platform is the bridge between users and the training platform,

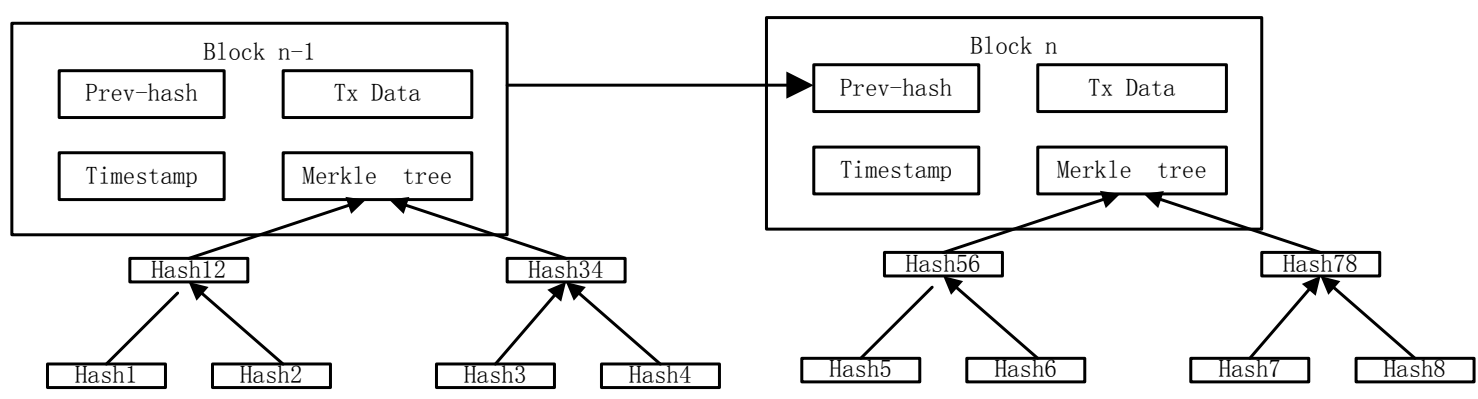

Figure 1. The basic structure of blockchain. 


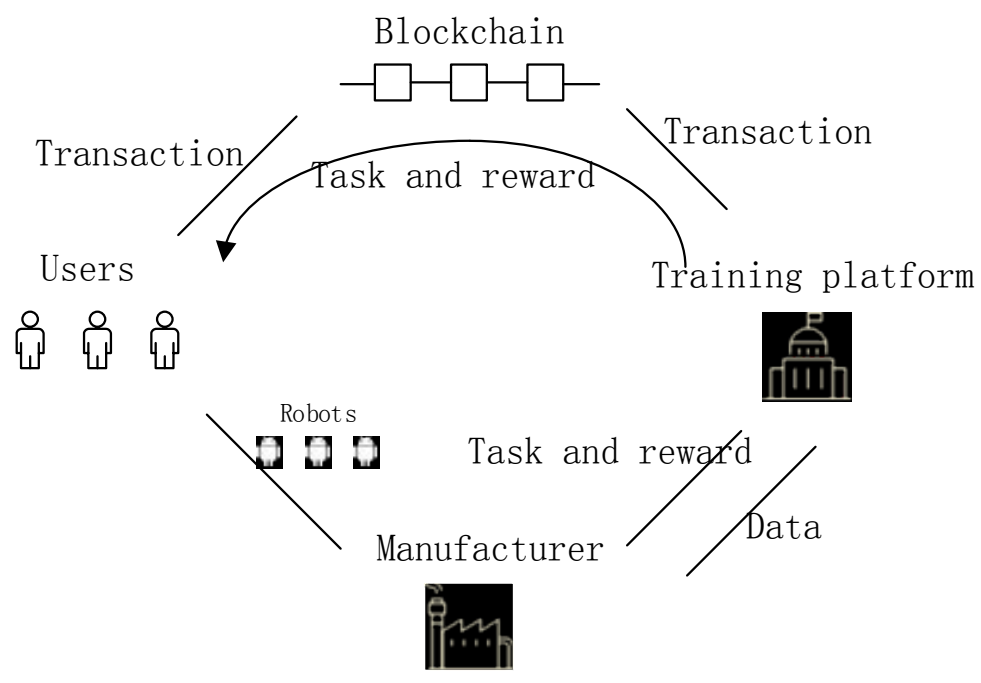

Figure 2. System model.

delivering tasks, rewards and training data. The attributes of task include task ID, specified training scene. For example, Hyperledger Fabric is an open source enterprise-grade permissioned distributed ledger technology platform. A Hyperledger Fabric channel is seen as a "subnet" for communication between some network participants for the purpose of privacy [12]. And there are some other open source blockchain platforms that can be used such as Monero [13].

Users get the task from training platform. They train the robot according to task requirements. After training, they upload the robot's training data to the blockchain to get rewards.

\section{Theoretical Analysis}

Each participating entity has its own expectations and benefits. Meanwhile they must provide services for the proposed system. Based on their expectations and benefits, we theoretically analyzed this system. They must follow the rules to get the benefits.

As shown in Table 1, robot manufacturers collect information about specific scenarios by issuing tasks and rewards. Those data can be used to train robots and improve robot performance. Training platform get handling fee from it. Users can train their own robots and choose whether to sell training data to the training platform to earn rewards. Blockchain platform can make users anonymous and protect users' privacy. The blockchain platform ensures the immutability and authenticity of data and protects the privacy of participants to a certain extent.

According to the rational people theory [14], everyone will choose the one that makes them more profitable. The assumption of rational people is the basic assumption in traditional economic theory. This assumption implies that anyone is a rational person, he is selfish, and wants to maximize his needs or desires. Therefore, the system we proposed is theoretically operational. 
Table 1. The entity's benefits from the system.

\begin{tabular}{|c|c|c|}
\hline Benefit & Want & Gain or not \\
\hline Manufacturers & Data & yes \\
\hline Training platform & Money & yes \\
\hline Users & Money & yes \\
\hline Blockchain & Money & yes \\
\hline
\end{tabular}

\section{Conclusion}

In this paper, firstly, we introduced the security and privacy issues in the process of intelligent robot learning. Then, we proposed a smart robot training data acquisition and learning process recording architecture based on blockchain to ensure that the training information of robot is kept credible and to provide users with privacy protection. However, we do not implement and analyze the proposed system. Future work includes a full-fledged system and implementing the system to test its performance in a real environment.

\section{Conflicts of Interest}

The authors declare no conflicts of interest regarding the publication of this paper.

\section{References}

[1] Bryndin, E. (2019) Social Cognitive Smart Robots: Guide, Seller, Lecturer, Vacuum Cleaner, Nurse, Volunteer, Security Guard, Administrator. Communications, 7, 6-12. https://doi.org/10.11648/j.com.20190701.12

[2] Goodrich, M.A. and Schultz, A.C. (2008) Human-Robot Interaction: A Survey. Now Publishers Inc., Hanover. https://doi.org/10.1561/9781601980939

[3] Chew, Y.J., Wong, K.S. and Ooi, S.Y. (2017) Privacy Protection in Machine Learning: With the State of the Art for Private Decision Tree. Security and Authentication: Perspectives, Management and Challenges. Nova Science Publishers, Inc., Hanover, 13-39.

[4] Bitcoin, N.S. (2019) A Peer-to-Peer Electronic Cash System. Manubot.

[5] Bitcoin, L.J. (2013) Theory, Practice and Effect. Studies of International Finance, 12, 14-25.

[6] Bonneau, J., Miller, A., Clark, J., et al. (2015) Sok: Research Perspectives and Challenges for Bitcoin and Cryptocurrencies. 2015 IEEE Symposium on Security and Privacy, San Jose, 17-21 May 2015, 104-121. https://doi.org/10.1109/SP.2015.14

[7] Bishop, G. (2017) Illinois Begins Pilot Project to Put Birth Certificates on Digital ledger Technology.

[8] Browne, R. (2017) IBM Partners with Nestle, Unilever and Other Food Giants to Trace Food Contamination with Blockchain. CNBC, 22 August 2017.

[9] Lakhani, K.R. and Iansiti, M. (2017) The Truth about Blockchain. Harvard Business Review, 95, 119-127.

[10] Simonsen, S. (2017) Reasons the UN Is Jumping on the Blockchain Bandwagon. 
[11] Wang, K., Liu, M., Jiang, X., et al. (2019) A Novel Vehicle Blockchain Model Based on Hyperledger Fabric for Vehicle Supply Chain Management. International Conference on Blockchain and Trustworthy Systems, Springer, Singapore, 732-739. https://doi.org/10.1007/978-981-15-2777-7_59

[12] Androulaki, E., Barger, A., Bortnikov, V., et al. (2018) Hyperledger Fabric: A Distributed Operating System For Permissioned Blockchains. Proceedings of the Thirteenth EuroSys Conference, Porto, April 2018, 1-15.

https://doi.org/10.1145/3190508.3190538

[13] Cao, T., Yu, J., Decouchant, J., et al. (2020) Exploring the Monero Peer-to-Peer Network. Financial Cryptography and Data Security 2020, Sabah, 10-14 February 2020, 578-594. https://doi.org/10.1007/978-3-030-51280-4_31

[14] Allingham, M. (2002) Choice Theory: A Very Short Introduction. OUP, Oxford. https://doi.org/10.1093/actrade/9780192803030.001.0001 